\title{
A Novel Cross-layer Framework For Large Scale Emergency Communications
}

\author{
Vu San Ha Huynh \\ School of Computer Science \\ University of Nottingham \\ United Kingdom \\ psxvsh@nottingham.ac.uk
}

\author{
Milena Radenkovic \\ School of Computer Science \\ University of Nottingham \\ United Kingdom \\ Milena.Radenkovic@nottingham.ac.uk
}

\begin{abstract}
This paper explores the problem of improving coverage and capacity of large-scale communication networks in disaster-struck areas. We propose a novel integrated dynamic cross-layer distributed energy aware emergency framework, $E^{3} \mathbf{F}$, that spans large geographical areas and variable time ranges. $E^{3} \mathbf{F}$ enables adaptive storage, dynamic packet scheduling and utility driven forwarding that avoids congestion and energy depletion. Our extensive experiments with realistic traces show significantly improved energy efficiency and low overheads while maintaining high success ratios for both data dissemination and query answering.
\end{abstract}

Keywords-Heterogeneous Opportunistic Networks, Emergency communications, Cross-layer design, Quality of Service.

\section{INTRODUCTION}

In the events of man-made or natural disasters, existing network infrastructures may become unstable, inaccessible, or unusable. Communication in such hostile conditions is usually faced with three causes of failure: physical destruction, lack of power supply and network congestion (i.e. as access to the infrastructure decreases, network become partitioned, signal strength decreases and regions of the network become overloaded due to sudden surges of traffic). Current solutions cannot cope with large-scale disaster scenarios. Emerging activities led by industry to alleviate post-disaster communications [13], such as Alcatel-Lucent Broadcast Message Centre, CISCO network emergency response vehicles or Ericsson WLAN emergency disaster response are short term solutions with short range coverage and are not applicable to isolated rural and large scale scenarios which are the focus of this paper.

We propose a novel unified framework which aims to overcome severely limited communications, be able to improve power at the remote network edges and support different types of emergency traffic and services. The use of MANETs and VANETs is not always feasible in large-scale emergencies whilst existing Opportunistic Networks, which allow nodes to communicate without the infrastructure, are typically not optimised for criteria needed for our scenarios such as energy harvesting and congestion avoidance. Therefore, we propose the Energy Efficient CongEstion Aware cross-layer intelligent Emergency Framework $\left(E^{3} F\right)$ that supports different types of traffic and services. $E^{3} \mathrm{~F}$ is selforganised and adaptive while allowing interaction with human users. $\mathrm{E}^{3} \mathrm{~F}$ runs in static and mobile devices (smart-phones, vehicles, etc) and establishes peer-to-peer communications for efficient data query and dissemination.

We evaluate our new framework using real vehicular GPS traces of fire engines from the north-west Spain [4]. Our core objective is improving the reliability and scalability of valuable messages about potential victims or cooperation emergency responses reaching their destination. As disasters may last arbitrarily long and the battery life of devices is limited, energy efficiency plays a vital role in our solution. [4] and [5] showed that this trace highly partitioned, sparse and dynamic. We evaluate $\mathrm{E}^{3} \mathrm{~F}$ performance against the state-ofthe-art emergency OppNet routing protocol [1] across multiple criteria such as message success ratio, delay, overhead and energy consumption. The contributions of this paper are multifold: we provide dramatically increased coverage by exploiting new energy polling algorithm, intelligent packet scheduling and adaptive forwarding mechanisms while keeping resource overhead low and supporting high success ratios for two types of traffic: ad hoc query and dissemination. The paper begins by providing an overview of the related work in section II, section III introduces energy efficient and congestion-aware emergency framework $\left(\mathrm{E}^{3} \mathrm{~F}\right)$ architecture, section IV evaluates $\mathrm{E}^{3} \mathrm{~F}^{\prime} \mathrm{s}$ performance and section $\mathrm{V}$ gives the conclusion.

\section{RELATED WORK}

Most research in resilient disaster communications covers networking solutions either describing the mobile response components, processes for restoring failed network cells or priority service functions in the public switched telephone networks using cellular networks after the disaster to rapidly bringing normal phone line back to the end users [13-16, 25]. However, these are standalone solutions which are applied to separated networks and cannot be used for communication in more heterogeneous disaster scenarios.

Opportunistic networks were first introduced in [17] in the context of Emergency Preparedness and Response (EPR). [1] evaluates a range of different opportunistic networks routing protocols: Epidemic, Prophet, MaxProp [2] and TTR [8] in disaster scenarios running by simulating mobility models. The authors argued that for varying number of nodes and data quantity, MaxProp achieves the best delivery performance 
while TTR has the lowest overhead ratio. In [3], two energy efficient OppNets routing protocols PropTTR and PropNTTR are proposed to balance the trade-off between MaxProp and TTR. Both of these, however, resulted in lower success ratios than MaxProp. [18] uses another cluster mobility model and argued that there is no best opportunistic protocol in terms of delivery performance for generic topologies and mobility models.

Vehicular clouds associated with their different unique features are overviewed in detail in [27]. The authors presented several emergent problems in vehicular cloud (e.g. cost of services, energy consumption, network congestion) which have not been solved due to, for example, lack of network resources, architecture scalability and heterogeneity. Our previous works $[11,26]$ describes low-cost personal clouds (deployed on Raspberry Pis, smart-phones) which can host, store and monitor a range of information and services locally while still being able to share with others. Note that the term "node" that we use in this paper can refer both vehicular cloud and mobile personal clouds without loss of generality. In order to overcome with large scalability, heterogeneous and unreliable networks, [9] presented an idea of utilising Distributed Hash Table (DHT) to propose a self-organised, 2P2 overlay for a storing large amount of data. We use this in the information centric layer of $\mathrm{E}^{3} \mathrm{~F}$.

To improve our quality of service, $\mathrm{E}^{3} \mathrm{~F}$ builds on $[2,20,28]$ in order to assign dynamic priorities to messages before forwarding. Similarly to [8], which takes advantage of short connectivity forwarding between nodes for the creation of electronic triage tags for delivering to a Coordination Point without communication infrastructure, we utilise heterogeneous localised communication. Building on our previous work, FDASS [19] which proposed an intelligent framework that enhances the reliability of the manufacturing plant dealing with varying network connectivity and nonuniform distribution of different types of faults in the network, E3F enables energy efficiency, large scale and mobility.

The GPS trace [4] was collected from the regional Fire Department located in Oviedo in northern Spain. The trace was generated by GPS devices embedded in vehicles, a helicopter and a few personal radios from the period between October 2011 and September 2012. Annual technical report about Forest Fires in Europe, Middle East and North Africa [10] show that October (2011) has the highest number of fires and burnt areas in Spain. Thus, we choose October 2011 as the most challenging period and explored communication feasibility in significantly sparser and larger contexts than the previous traces. [5] showed that some vehicles in this trace were always in car park and either made long contacts with others or were isolated. Therefore, we found and removed those nodes from the data trace to avoid the bias in our experiments. [23] deployed over a similar trace an overlay routing protocol for video over sparse MANETs achieving below $50 \%$ success ratios which are critically low rates while this paper shows that $\mathrm{E}^{3} \mathrm{~F}$ manages around $80 \%$.We briefly compare Oviedo trace [4] to another well-known sparse vehicular data trace, San Francisco Cab [24] and show several key parameters in Table 1.

\begin{tabular}{|c|c|c|}
\hline Parameter & Oviedo & San Francisco \\
\hline Simulation time & 7 days & 1 month \\
\hline \# of nodes & 126 & 100 \\
\hline Communication range & $50 \mathrm{~m}$ & $50 \mathrm{~m}$ \\
\hline Area & $\approx 10000 \mathrm{~km}^{2}$ & $\approx 100 \mathrm{~km}^{2}$ \\
\hline Average number of contacts & 1150.5 & 7241.5 \\
\hline
\end{tabular}

Table 1. Values of the parameters

\section{ENERGY EFFICIENT AND CONGESTION AWARE EMERGENCY FRAMEWORK $\left(\mathrm{E}^{3} \mathrm{~F}\right)$}

\section{A. Overview}

In this section, we describe an innovative cross-layer framework $E^{3} \mathrm{~F}$ that supports energy polling, congestion avoidance, self-organised communication, information-centric data dissemination and query answering. We identify inherent limitations in the trade-off between power and data transfer and aim to avoid emergency bottlenecks through both packet scheduling and smart multi-hop forwarding. $\mathrm{E}^{3} \mathrm{~F}$ manages to reduce energy consumption and avoid depletion of more important nodes.

$E^{3} \mathrm{~F}$ consists of 3 classes of nodes: i) Internal nodes which are inside emergency areas (e.g. victims or firefighters). These nodes have resource constraints (low bandwidth, speed, range coverage, etc), thus can store, forward and disseminate a limited number of messages. ii) Fire vehicles which have more powerful resources and can store their own state data in addition to replicating other nodes' data, send and reply to query messages. iii) External Helper nodes which could be powerful devices deployed on demand in a particular emergency scenario. These nodes have more resources than vehicles (e.g. range), offering more bandwidth and connectivity. These 3 types of nodes may be upgraded or downgraded depending on their resources and availabilities.

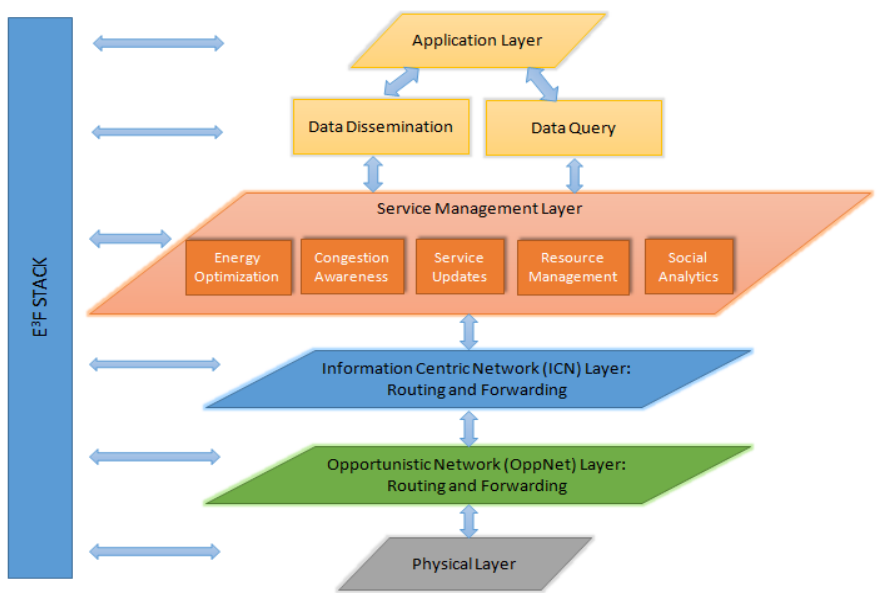

Figure 1. Energy Efficient and Congestion Aware Emergency Framework Cross-layer Architecture $\left(\mathrm{E}^{3} \mathrm{~F}\right)$

Fig. 1 describes $E^{3} F$ cross-layer architecture which consists of multiple layers including: Physical Layer, Opportunistic Network Layer, Information Centric Network Layer, Service Management Layer, Traffic and Application Layer. 
Application Layer in $\mathrm{E}^{3} \mathrm{~F}$ offers 2 types of services: data dissemination and multi-attribute data query.

In the Opportunistic Network Layer, nodes scan and discover neighbours before exchanging encountered information in multi-dimensional vectors. The exchanged information includes a list of neighbours, node encountered history, remaining buffer level analysis and remaining energy levels. Similarly to FDASS [19], the Opportunistic Network Layer enables predictive analytics of nodes' connectivity and availability using localised network communication.

The Information Centric Network Layer allows 2 types of traffic: dissemination and query answering, acquires the context of nodes (e.g. location, tag, node type) by defining new $E^{3} F$ packet format given in Fig. 2. Aligned to [9], data naming is hashed and looked up in Distributed Hash Table (DHT). $\mathrm{E}^{3} \mathrm{~F}$ associates it with data content to form key-value pairs.

\begin{tabular}{|c|c|c|c|c|}
\hline \multirow{3}{*}{ Header } & Source ID & $\begin{array}{c}\text { Destination ID } \\
\text { (Optional) }\end{array}$ & Time & $\begin{array}{c}\text { Location } \\
\text { (Optional) }\end{array}$ \\
\cline { 2 - 5 } & Packet Type & Packet Priority & Tag \\
\cline { 2 - 5 } & Node Type & Data Offset & Checksum \\
\hline \multirow{2}{*}{ Body } & List of contacts & Number of hops & Padding \\
\cline { 2 - 4 } & \multicolumn{3}{|c|}{ Contacts state and application Data } \\
\hline
\end{tabular}

Figure 2. $\mathrm{E}^{3} \mathrm{~F}$ packet format.

In the Service Management Layer, there are five integrated modules: Resource Management, Social Analytics, Congestion Awareness, Energy Optimisation and Service Updates. Resource Management module allows real-time predictive buffer storage, intelligently schedules and prioritises packets (e.g. deciding which packets should be transmitted first and which ones should be deleted from the buffer); Social Analytics module examines social metrics (similarity, betweeness, tie strength) of neighbours and calculates its social heuristic utility value in order to find the potential best next hop; Congestion Awareness module measures the retentiveness and receptiveness of nodes to avoid overload and congestion in high-utility-value nodes; Energy Optimisation module differentiates preserved battery levels for different types of nodes (e.g. higher priority to preserve battery level of more important nodes), measures the current levels of battery capacity and calculates the energy cost of all paths, to make final forwarding decisions. The Service Updates module handles 2 types of packet: data dissemination and query answering.

\section{B. Service Management Layer}

This section presents the Service Management Layer components in more details.

Resource Management Module: $\mathrm{E}^{3} \mathrm{~F}$ initializes a priority value $\mathrm{p}_{0}$ for each new packet which may subsequently change as the packet traverses the network:

$$
\mathrm{p}_{\mathrm{i}}=\alpha * \mathrm{p}_{\mathrm{i}-1}
$$

in which the priority of the packet in node $\mathrm{i}\left(\mathrm{p}_{\mathrm{i}}\right)$ depends on its priority in the previous hop $\left(\mathrm{p}_{\mathrm{i}-1}\right)$ multiplied by the willingness $\alpha$ of node $i$ to forward the packet. $\alpha$ varies from 0 (if node $p$ has critical low battery level, for example) to the maximum (MAX) number.
After setting up initial priority, we calculate packet delivery likelihood based on MaxProp [3] technique. A node $\mathrm{i}$ $\in \mathrm{N}$ has a probability $f_{j}^{i}$ to meet node $\mathrm{j} \in \mathrm{N}$. Initially, $f_{j}^{i}=$ $\frac{1}{|N|+1}$. When node $\mathrm{i}$ encounter node $\mathrm{j}, f_{j}^{i}:=f_{j}^{i}+1$ and all other $f^{i}$ will be re-normalized. The cost of packet delivery from node $\mathrm{i}$ to destination $\mathrm{d}$ is:

$$
\mathrm{c}(\mathrm{i}, \mathrm{i}+1, \ldots, \mathrm{d})=\sum_{i}^{d-1} 1-f_{i+1}^{i}
$$

If the path from node $i$ to destination $d$ is unknown, $\mathrm{c}$ is set to infinite.

$\mathrm{E}^{3} \mathrm{~F}$ combines initial packet priority with dynamic delivery likelihood to calculate the final priority value of the packet. Packets with the lowest priority are deleted from the buffer first, packets with the highest priority will be examined further to make forwarding decisions.

Social Analytics Module: $\mathrm{E}^{3} \mathrm{~F}$ does not flood high priority packets in the network. The Social Analytics module calculates a heuristic utility value of all neighbours based on similarity, betweeness and tie strength metrics (which we define differently to how it has been defined in [12]) in order to find the best next hop for forwarding packets.

Util(j) $=\alpha * \operatorname{Similarity}()+\beta *$ Betweeness ()$+\gamma^{*}$ Tie Strength ()

$\alpha+\beta+\gamma=1$

Each packet header has a location and tag field. When node $\mathrm{j}$ is in the same region or has the same tag with destination d, it will be given: $\operatorname{Similarity}()=1$. Otherwise, similarity will be given as the number of common neighbours between 2 nodes:

$\operatorname{Sim}(i, d)=|N(i) \cap N(d)|$

Similarity ()$=\frac{\operatorname{Sim}(j, d)}{\operatorname{Sim}(i, d)+\operatorname{Sim}(j, d)}=\frac{|N(j) \cap N(d)|}{|N(i) \cap N(d)|+|N(j) \cap N(d)|}$

We calculate betweeness and tie strength using [12] techniques. As $\mathrm{E}^{3} \mathrm{~F}$ assigns adaptively weight $\alpha, \beta, \gamma$ to $\operatorname{Sim}()$, $\operatorname{Bet}()$ and $\mathrm{TS}()$ respectively and we argue that in emergency scenarios, similarity metric is more important than the other two, thus: $\alpha>\beta+\gamma$. In our experiment, we set $\alpha=0.8, \beta=\gamma=$ 0.1

For Tie Strength metric, we favour weak-strength tie in order to allow packets traverse in the network as far as possible.

Congestion Awareness Module: The above two subsections describe core part of $\mathrm{E}^{3} \mathrm{~F}$ that decides which packets to be transmitted and which neighbours to be the next hops for forwarding packets. However, overusing a high-utility-value node will make it congested, results in the congestion in part of the network or even the whole network. Furthermore, overload in a node may cause extremely quick battery drainage and make the node totally unusable. We apply our previous works, CAFE [22] and CafRep [6], for $\mathrm{E}^{3} \mathrm{~F}$ in order to avoid the use of nodes that have high social-utility-value but less availability and encourage using nodes with higher level of buffer. The Congestion Awareness calculates Retentiveness Utility (as contact's remaining buffer) and Receptiveness Utility (as average delay a node has added to the packets it has held) in order to redirect the traffic to less congestion part of the network. 
Energy Optimisation Module: Building on our previous work [7], the Energy optimisation module in Service Management Layer gets remaining energy level $\mathrm{E}$ of neighbours to avoid forwarding packets to nodes with high availability but has lower battery capacity. Remaining lifetime $\mathrm{T}$ of a node is $\mathrm{Ti}=\frac{E i}{C i}$ in which $\mathrm{C}$ is the energy cost to transmit a message. $\mathrm{E}^{3} \mathrm{~F}$ uses the next hop with $\max (\mathrm{T})$. As we use the same energy cost $\mathrm{C}$ for every type of message, $\mathrm{E}^{3} \mathrm{~F}$ chooses the hop with $\max (\gamma * \mathrm{E})$ in which $\gamma$ is the threshold specified for different types of nodes. The $\gamma$ will be set to be low if a node is an important node which has to be protected from being battery drainage.

Service Updates Module: The Service Updates module allows $E^{3} \mathrm{~F}$ to deal with different types of traffic: data dissemination and data query. When a node creates a message either for dissemination or query, the following steps are performed: firstly, the Service Updates module generates data content as format shown in Fig. 2. After that, the recipients of a packet are determined (Fig. 3) by getting either destination ID or data content (Information-centric network). If the hopcount threshold $\mathrm{N}$ is set up, the packet is allowed to traverse up to $\mathrm{N}$ hops before being removed from the network regardless of whether it is successfully disseminated to all interested nodes (for dissemination) or whether it reaches a node which has an answer (for query). In emergency scenarios, that may avoid long response when a packet traverses too far from the source. Finally, the Service Updates module selects best next-hops from candidate paths (Fig. 4) using utility value collected from the other 4 modules mentioned above.

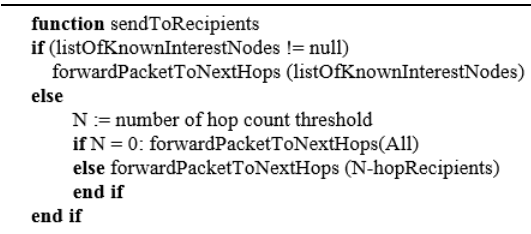

Figure 3. Determining recipients.

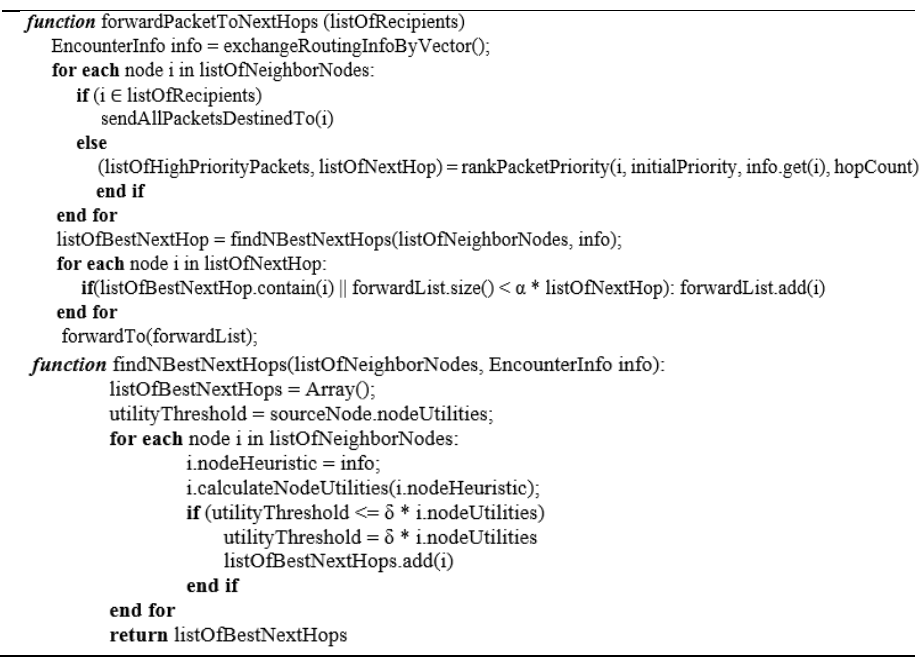

Figure 4. Selecting best next-hops from candidate paths.

\section{EVALUATION}

In this section, we present and evaluate the performance of $\mathrm{E}^{3} \mathrm{~F}$ by comparing it with the state-of-the-art OppNets routing protocol, MaxProp tailored for emergency scenarios which we refer to EmeMaxProp. We run the ONE simulation [21] with real Oviedo emergency data trace [4]. The simulation parameters details are shown in Table 2.

\begin{tabular}{|l|c|c|}
\hline \multirow{4}{*}{ Mobility } & Parameter & Value \\
\hline Network & Disaster Area & $\approx 225 \mathrm{~km}^{2}$ \\
\cline { 2 - 3 } & \# of original nodes & 37 \\
\cline { 2 - 3 } & \# of external Helper nodes & $0-5$ \\
\cline { 2 - 3 } & \# of internal nodes & $0-40$ \\
\cline { 2 - 3 } & Simulation time & 2 weeks \\
\cline { 2 - 3 } & Link speed & Internal nodes: $6.75 \mathrm{MBps}$ \\
\cline { 2 - 3 } & & External nodes: $8.75 \mathrm{MBps}$ \\
\cline { 2 - 3 } & Radio range & Internal nodes: $50 \mathrm{~m}$ \\
\cline { 2 - 3 } & & External vehicles: $200 \mathrm{~m}$ \\
\cline { 2 - 3 } & & External Helper nodes: $5 \mathrm{~km}$ \\
\cline { 2 - 3 } & & External nodes: $\geq 10 \mathrm{~GB}$ \\
\cline { 2 - 3 } & Message size & $1 \mathrm{MB}$ \\
\cline { 2 - 3 } & TTL & 1 week \\
\cline { 2 - 3 } & Message generation rate & 1 message per 15 minutes \\
\hline MaxProp & Meeting Prop Set Max Size & 50 \\
\hline & $\alpha$ & 1 \\
\hline
\end{tabular}

Table 2. Values of the simulation parameters.

\section{A. Evaluation in the presence of dynamic number of external Helper nodes}

Apart from vehicles from the original data trace, we added a variable number of external Helper nodes with higher availability. These nodes may include Access Points, temporary military emergency infrastructure or state infrastructure devices. We evaluate $\mathrm{E}^{3} \mathrm{~F}$ against EmeMaxProp with and without node energy constraints. Figure 5 shows that the success ratio is increasing with the higher number of external nodes for both data dissemination and data query. We also observe that $\mathrm{E}^{3} \mathrm{~F}$ gives equal or better success ratio compared to EmeMaxProp e.g $\mathrm{E}^{3} \mathrm{~F}$ achieves around $80 \%$ success ratio if there are 5 external Helper nodes. We also show that $E^{3} \mathrm{~F}$ does not significantly reduce success ratio compared to EmeMaxProp when we run EmeMaxProp without energy constraints (i.e. EmeMaxProp without energy constraints gets up to $87 \%$ for data dissemination and $82 \%$ for data query if there are 5 external Helper nodes added).

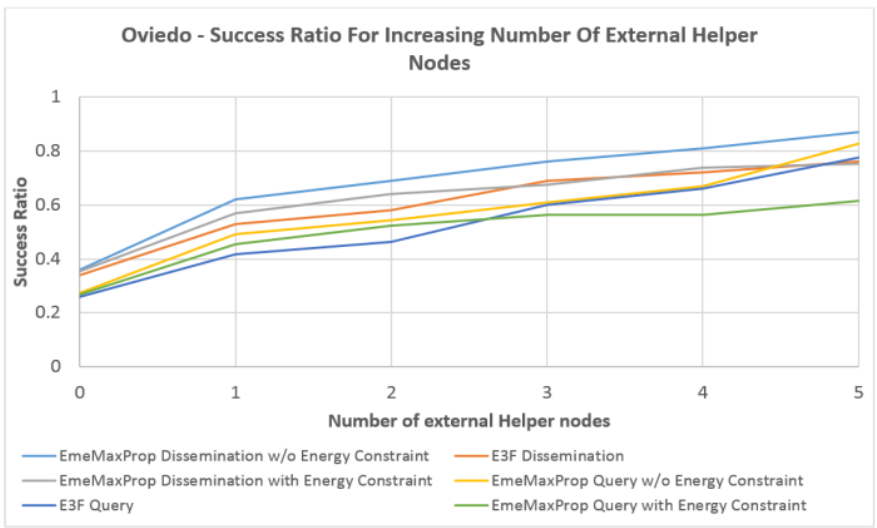

Figure 5. Success ratio and number of external Helper nodes 


\begin{tabular}{|c|c|c|}
\hline Type of nodes & $\begin{array}{c}\text { \% depleted nodes } \\
\text { EmeMaxProp }\end{array}$ & $\begin{array}{c}\text { \% depleted nodes } \\
\mathbf{E}^{\mathbf{3}} \mathbf{F}\end{array}$ \\
\hline Internal nodes & $40 \%$ & $10 \%$ \\
\hline Vehicles & $20 \%$ & $6.67 \%$ \\
\hline External Helper nodes & $50 \%$ & $0 \%$ \\
\hline
\end{tabular}

Table 3. Percentage of depleted nodes

Table 3 explains the decrease in delivery performance of EmeMaxProp if we limit the energy level of nodes. After 2 weeks of simulation, approximately $40 \%$ of internal nodes and $20 \%$ of vehicle nodes running EmeMaxProp are depleted due to battery drainage which also means messages would never deliver to destination. More importantly, over half of the external Helper nodes are depleted which is extremely unexpected because we argue that in the emergency scenarios, a node with high availability (high communication range, link speed, etc) should be protected from running out of energy. In contrast, after the duration of 2 weeks, $E^{3} \mathrm{~F}$ has no depleted external Helper nodes, while keeping the number of active nodes as high as possible $(90 \%$ internal nodes and $93.3 \%$ of vehicles nodes).

We evaluate the average energy consumption of $E^{3} F$ and EmeMaxProp while increasing number of external Helper nodes. Again, as expected, a higher number of external Helper nodes may help increase the delivery performance, but result in more involved hops which in turn, increase the average energy consumption. As shown in Fig.6, data query consumes much higher energy compared to data dissemination. It could be explained by the fact that data query takes more hops and time to reach the node who has the answer.

For both data dissemination and data query, $E^{3} F$ consumed as half average energy as EmeMaxProp. In data dissemination, when increasing number of external Helper nodes from 0 to 5 , the average energy consumption of $E^{3} \mathrm{~F}$ increases slightly from $2329 \mathrm{~J}$ to $3189 \mathrm{~J}$ while that of EmeMaxProp are $3024 \mathrm{~J}$ and $6095 \mathrm{~J}$, respectively. In data query, average energy consumption of $\mathrm{E}^{3} \mathrm{~F}$ increases by $2332 \mathrm{~J}$ whilst that of EmeMaxProp is $6119 \mathrm{~J}$.

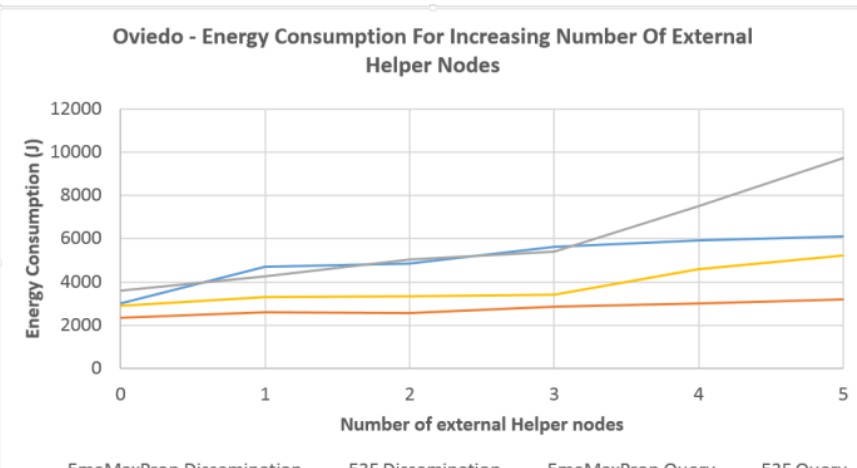

Figure 6. Average energy consumption and number of external Helper nodes

We consider overhead ratio to be the average number of replicas required to perform a successful delivery. While the overhead ratio of EmeMaxProp increases with respect to the growth of external Helper nodes, $\mathrm{E}^{3} \mathrm{~F}$ shows the decrease in the overhead ratio for both data dissemination and data query. The overhead ratio of $\mathrm{E}^{3} \mathrm{~F}$ is approximately $200 \%$ lower than EmeMaxProp (Fig. 7).

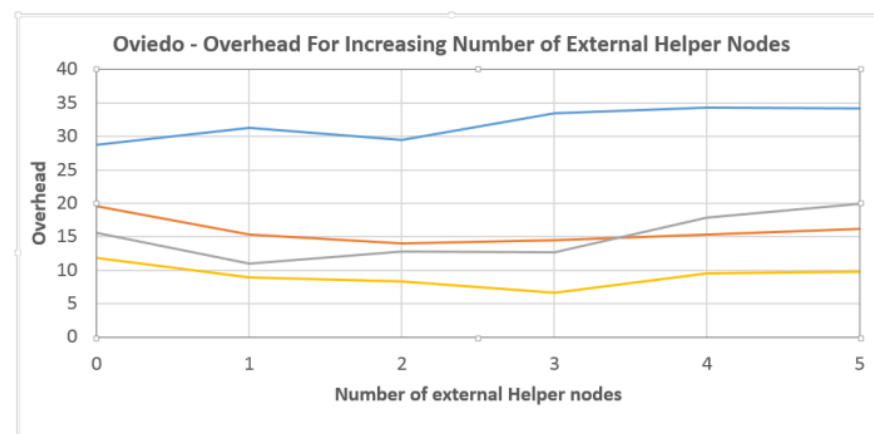

Figure 7. Overhead ratio and number of external Helper nodes.

\section{B. Evaluation in the presence of dynamic number of internal heterogeneous nodes}

In this section, we evaluate $\mathrm{E}^{3} \mathrm{~F}$ performance against EmeMaxProp for varying the number of internal heterogeneous nodes (such as people or firefighters who are inside emergency area) and in the presence of on one external Helper node with message generate rate of one message per hour.

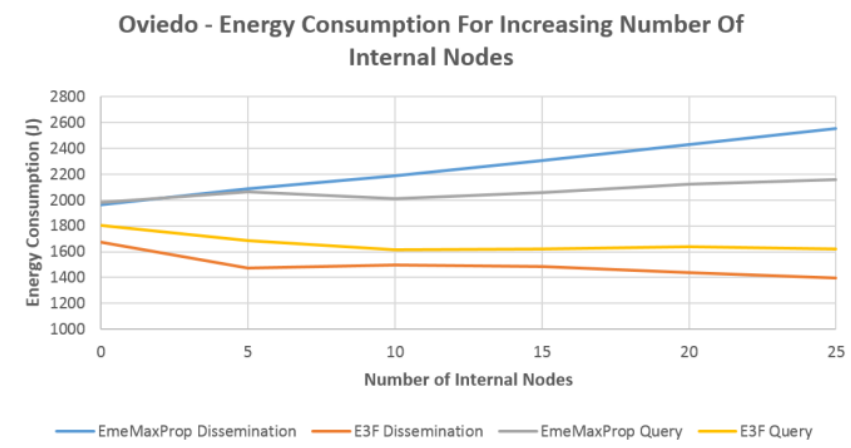

Figure 8. Average Energy Consumption and number of internal nodes.

In Fig 8, we show that $\mathrm{E}^{3} \mathrm{~F}$ consumes less $200 \%$ energy than EmeMaxProp for both data dissemination and data query. $\mathrm{E}^{3} \mathrm{~F}$ shows a decrease in energy consumption with respect to the increase in the number of internal nodes while the average energy consumption of EmeMaxProp increases as the number of internal nodes get larger, This is due to the higher number of nodes offering more forwaring opportunities for sending queries and getting answers. Due to the limited space, we are not able to show all results with our extensive network parameters but plan to do that in detail in the future publications.

\section{CONCLUSION}

We propose a novel, self-organised and adaptive Energy Efficient, CongEstion Aware cross-layer intelligent Emergency Framework $\left(\mathrm{E}^{3} \mathrm{~F}\right) . \quad \mathrm{E}^{3} \mathrm{~F}$ enables packet prioritisation and buffer management, applies complex 
temporal network centrality analytics and other relative utility functions to avoid congestion and make intelligent forwarding decisions. $\mathrm{E}^{3} \mathrm{~F}$ shows high message delivery performance for two types of traffic: data dissemination and query answering while keeping energy consumption low and adaptively preserve battery life for more important agents. In our future work, we plan to integrate $\mathrm{E}^{3} \mathrm{~F}$ to our low-cost prototyping testbed $[11,26]$ for more accurate performance validation. In addition, we will aim to extend $\mathrm{E}^{3} \mathrm{~F}$ to allow collaborations and cooperations between multiple vehicular clouds in order to enhance its performance. $\mathrm{E}^{3} \mathrm{~F}$ also should be able to interact with conventional cloud in less-challenging emergency scenarios.

\section{ACKNOWLEDGMENTS}

This work was supported in part by the FP7 "SelSus: Health Monitoring and Life-Long Capability Management for SELfSUStaining Manufacturing Systems" project, 609382.

\section{REFERENCES}

1. A. Martin-Campillo, J. Crowcroft, E. Yoneki, R. Mart. Evaluating opportunistic networks in disaster scenarios. Journal of Network and Computer Applications 36 (2), p.870-880, 2013.

2. J. Burgess, B. Gallagher, D. Jensen, and B. N. Levine. Maxprop: Routing for vehicle-based disruption-tolerant networking. Proceedings of IEEE INFOCOM, 2006.

3. A. Martín-Campillo and R. Martí. Energy-efficient forwarding mechanism for wireless opportunistic networks in emergency scenarios. Computer Communications, v.35, p.1715-1724. 2012.

4. S. Cabrero, R. García, X. G. García, D. Melendi, CRAWDAD dataset oviedo/asturies-er (v. 2016-08-08), downloaded from http://crawdad.org/oviedo/asturies-er/20160808, https://doi.org/10.15783/C7302B, 2016.

5. S. Cabrero, R. García, G. Paneda, D. Melendi. Understanding Opportunistic Networking for Emergency Services: Analysis of One Year of GPS Traces. Proceeding CHANTS '15 Proceedings of the 10th ACM MobiCom Workshop on Challenged Networks, p. 31-36, 2015.

6. M. Radenkovic, and A. Grundy. Efficient and Adaptive Congestion Control for Heterogeneous Delay Tolerant Networks. Ad Hoc Networks, v.10, n.7, p.1322-1345, 2012.

7. Wietrzyk, B., Radenkovic, M. Energy efficiency in the mobile ad hoc networking approach to monitoring farm animals. Sixth International Conference on Networking, 2007.

8. Martin-Campillo A, Crowcroft J, Yoneki E, Marti R, Martinez C. Using haggle to create an electronic triage tag. In: The second international workshop on mobile opportunistic networking ACM/SIGMOBILE MobiOpp, ACM Press p.167-170, 2010.

9. B. Wietrzyk and M. Radenkovic. Enabling Rapid and Costeffective Creation of Massive Pervasive Games in Very Unstable Environments. IEEE/IFIP WONS 2007.

10. European Commission, Joint Research Centre, DirectorateGeneral for the Environment. Forest fires in Europe, Middle East and North Africa 2011. European Commission, p.17-21, 2011.

11. M. Radenkovic, VSH Huynh. Low-cost Mobile Personal Cloud. Wireless Communications and Mobile Computing Conference (IWCMC), 2016 International p. 836-841, 2016.

12. E. M. Daly and M. Haahr. Social network analysis for information flow in disconnected delay-tolerant MANETs. IEEE Trans. Mobile Computing, v.8, n.5, p.606-621, 2009.
13. Mirnada, A Moloinaro, T Razafindralambo, A survey of Rapidly Deployable solutions for post disaster networks. IEEE Communication Mag, v.54, n.4, p.117-123, 2016.

14. N.-S. Vo, T. Duong, H.-J. Zepernick, and M. Fiedler. A crosslayer optimised scheme and its application in mobile multimedia networks with QoS provision. IEEE Syst. J., n.99, p.1-14, 2015.

15. H Zhang, C Jiang, Q Hu, Yi Quinm. Self-organisation in heterogeneous small cell networks. IEEE Networks, 2016

16. Al-Hourani, S. Kandeepan, A. Jamalipour. Stochastic geometry study on D2D communication as a disaster relief solution. IEE Transaction on Vehicle Technology, v.65, p.3005-3017. 2016.

17. Lilien, L., Gupta, A., \& Yang, Z. Opportunistic networks for emergency applications and their standard implementation framework. Performance, Computing, and Communications Conference, USA. p.588-593, 2007.

18. S. Saha, A. Sheldekar, C. R. Joseph, A. Mukherjee, and S. Nandi. Post disaster management using delay tolerant network. Communications in Computer and Information Science, v.162, p. 170-184, 2011.

19. M. Radenkovic, I. Kostadinov and B. Wietrzyk. Increasing Communication Reliability in Manufacturing Environments. Proc. IEEE IWCMC, Croatia. p. 1377-1383, 2015.

20. J. Inwhee and K. Sang-Bo. A Message Priority Routing Protocol for Delay Tolerant Networks (DTN) in Disaster Areas. International Conference on Future Generation Information Technology, p. $727-737,2010$.

21. A. Keränen, J. Ott and T. Kärkkäinen: The ONE Simulator for DTN Protocol Evaluation. SIMUTools'09: 2nd International Conference on Simulation Tools and Techniques. Rome. 2009

22. M Radenkovic, A Grundy. Congestion aware data dissemination in social opportunistic networks. ACM SIGMOBILE Mobile Computing and Communications Review 14 (3), p.31-33, 2010.

23. Cabrero S., G. Paneda, X., Plagemann, T., Melendi, D., Garcia, R. Towards reliable video transmission over sparse MANETs in emergencies. Informatica Na Educacao: Teoria \& Prática, 2011.

24. M. Piorkowski, N. Sarafijanovic-Djukic, M. Grossglauser, CRAWDAD dataset epfl/mobility (v. 2009-02-24), downloaded from http://crawdad.org/epfl/mobility/20090224, https://doi.org/10.15783/C7J010, Feb 2009

25. J. Zhang; A. Marshall; R. Woods; T. Q. Duong. Design of an OFDM Physical Layer Encryption Scheme. IEEE Transactions on Vehicular Technology, v, n.99, p.1-1, doi: 10.1109/TVT.2016.2571264

26. Milena Radenkovic, Jon Crowcroft, Mubashir Husain Rehmani: Towards Low Cost Prototyping of Mobile Opportunistic Disconnection Tolerant Networks and Systems. IEEE Access 4: p.5309-5321, 2016.

27. T. Mekki, I. Jabri, A. Rachedi, M. Jemaa. Vehicular Cloud Network: Challenges, Architectures, and Future Directions. Vehicular Communications, doi: 10.1016/j.vehcom.2016.11.009. 2017.

28. A. Takahashi, H. Nishiyama, and N. Kato. Fairness issue in message delivery in delay- and disruption-tolerant networks for disaster areas. Proceedings of the International Conference on Computing, Networking and Communications, USA. p.890-894, 2013. 